\title{
IMPROVED ASSESSMENT OF DELAYED NEUTRON DETECTOR DATA IN CANDU REACTORS
}

\author{
Will Aylward \\ University of Strathclyde \\ Glasgow, Scotland, UK \\ Graeme West \\ University of Strathclyde \\ Glasgow, Scotland, UK
}

\author{
Christopher Wallace \\ University of Strathclyde \\ Glasgow, Scotland, UK
}

\author{
Curtis McEwan \\ Bruce Power \\ Tiverton, Ontario, Canada
}

Keywords: CANDU, Fuel defects, Monitoring, Decision Support, Machine Learning,

\begin{abstract}
A common challenge at nuclear power plants is to ensure that routinely-collected data is fully utilised. Data analytics provides an opportunity for improvements in prognostics and health monitoring by identifying correlations in related datasets without major capital investment.

This paper describes work focused on improving the fuel defect identification process in Bruce Power's eight CANDU nuclear reactors in the province of Ontario, Canada. The CANDU reactor comprises individually-pressurised horizontal channels which can be refuelled without taking the reactor offline. The detection and location of fuel defects is typically achieved using two systems: the first monitors the primary coolant for the presence of fission products and specific radionuclides, and is used to detect the presence of fuel defects within the core. The second system is deployed periodically and uses the emission of delayed neutrons to identify the channel containing defect fuel.

In this paper we focus on improving the assessment of online delayed neutron monitoring data, with the aim of reducing the time period between initial detection of an in-core defect to identification and removal of the damaged fuel. The existing process is manually intensive, reliant on the judgement of a domain expert and operating experience demonstrates that this time period varies considerably.

A first stage of investigation examines potential improvements to the current data processing system: developing and applying new analytic techniques in this area has shown promising results, with some fuel defects potentially identified several days earlier than the current system. Results from some short representative case studies are presented and further work will consider a larger volume of data.
\end{abstract}

In addition, an extensive historical dataset is available which spans several years. In the second stage of investigation, the paper explores previously undocumented trends in this data and discusses the potential to produce correlations with other reactor parameters. The application of this knowledge can lead to opportunities in the use of Machine Learning algorithms to allow more accurate predictions to be made.

\section{INTRODUCTION}

Data is constantly gathered at nuclear power plants, and is generally used to help engineers and operators make key decisions regarding specific plant operations. While operators will have a deep technical knowledge of the systems they are managing, there is often limited re-use or statistical analysis of data once it has served its initial purpose. As a result of advances in computer-driven machine learning which have not yet been fully leveraged in many operational areas, there exists an opportunity to improve the decision making process so that faults may be detected earlier, more accurately and with fewer dedicated personnel hours. These advances can also enable larger historical datasets to be leveraged, and combining this with novel analytics can lead to a deeper understanding of the core behaviour at little extra cost to plant operators.

Here we present the initial assessment and analysis of data from one such area: that of delayed neutron monitoring for fuel defect detection in CANDU nuclear plants.

\section{BACKGROUND}

\subsection{CANDU Reactors}

Bruce Power operates eight CANDU nuclear reactors in the province of Ontario, Canada. Fuel in the CANDU reactor is arranged in bundles, each containing 37 fuel elements, with 12 bundles in each of the 480 pressure tubes. One advantage of the design is that every fuel channel can be individually refuelled without taking the reactor offline. (Garland et al., 2014). 


\subsection{Motivation for fuel monitoring}

Components within CANDU reactor cores are subject to high levels of stress due to a number of factors. The primary source of damage is the intensity of heat created in the fission process, but the heat fluctuation and high flow speed of the heavy water coolant are also major contributors.

Fuel elements comprise a number of uranium oxide pellets contained within a thin zircalloy sheath. Ongoing exposure to fast neutrons causes embrittlement and weakness of this sheath. Upon refuelling, the coolant is allowed to carry the new fuel bundle into the reactor, resulting in unavoidable collisions between new and old fuel bundles, leading to another source of materials stress (Garland et al., 2014).

As a result of the stresses the fuel is exposed to, defects can occasionally occur. The frequency of events such as these in CANDU reactors are some of the world's lowest amongst water cooled reactors (IAEA, 2010), but it remains important that they are detected and removed quickly.

When a fuel defect occurs, fission byproducts are able to escape into the coolant. Some of these byproducts have long half-lives and can cause long term contamination of the primary coolant loop, which has specific operating limits for radiation levels (AECL CANDU, 1993). Fuel bundles with multiple defects are more difficult to remove from the reactor, which further incentivises the identification and location of defects at an early stage.

\subsection{Fuel Monitoring Systems}

The detection and location of fuel defects in CANDU reactors is achieved on some sites using two systems: the first monitors the primary coolant for the presence of fission products and specific radionuclides and is used to indicate the presence of defect fuel somewhere within the core. The second system is deployed periodically and uses the emission of delayed neutrons (DNs) to identify the channel containing defect fuel. The data generated by this second system is the focus of the work presented here, but the information derived from the GFP system is useful when interpreting the DN data and will be referenced later.

If a defect occurs, DNs are released and detectable in monitoring halls outside the reactor, predominantly via $\mathrm{I}^{137}$ with half-life of 22 seconds and $\mathrm{B}^{87}$ with half-life of 56 seconds (AECL CANDU, 1993). Inside the reactor, DNs from escaped fission products make up only approximately $3 \%$ of total activity, however a short time after leaving the core they account for $75 \%$ of DN activity due to the longer half-lives of these specific fission byproducts. As a result, it is possible to detect which channel contains a fuel defect by measuring neutron activity levels.

The 480 channels are monitored via two measurement halls; one at each end of the reactor. Sample tubes for all 480 channels are connected to either hall, designed in order to allow a transit time between reactor and measurement hall of 45 seconds which allows the defect fuel to be identified as outlined above. Some differences may arise in this transit time figure due to system pressure deviations, pressure loss calculation errors for pipe fittings, and in-line coolant boiling.

Measurement points for each of the 240 channels are arranged in an 8x30 array in each measurement hall. These measurement points are surrounded by tanks of demineralized water to ensure isolation of readings. A rig comprising 8 neutron detectors moves horizontally across this array, stopping for a short period at each sample point to simultaneously collect 8 neutron count values; originally this period was one minute (Lipsett \& Tseng, 1976) but was subsequently lengthened to 90 seconds. The collection of DN activity data for all 240 channels in a single session in a single hall is now referred to as a 'scan'.

After the coolant has passed through the measurement hall, it is returned to the reactor and recirculates. As a result, elevated DN readings are initially observed for just one channel but subsequently all channels will exhibit higher counts, although the channel containing defective fuel should continue to display the highest counts (AECL CANDU, 1993).

\subsection{Existing Analysis Process}

Bruce Power employs a Double Normalisation $(2 \mathrm{xN})$ technique to allow comparison and identification of the channel(s) with defective fuel. For each detector, count values are first filtered then normalized with respect to the average count rate for the 30 channels in question. This removes most effects of any settings changes between scans, and goes some way towards removing any inter-monitor bias, assuming all monitors are connected to similarly active channels. Secondly, data is filtered and normalized channelwise, so if one channel consistently produces a higher reading than others, this bias will be removed (Schmeing \& Hogg, 2005).

This algorithm is applied to every group of channels for all 8 detectors, and by plotting these values any anomalous channels can be identified as their double normalized counts will usually appear to trend higher than their neighbours. Fig. 1 shows an example of data from a group of channels, with

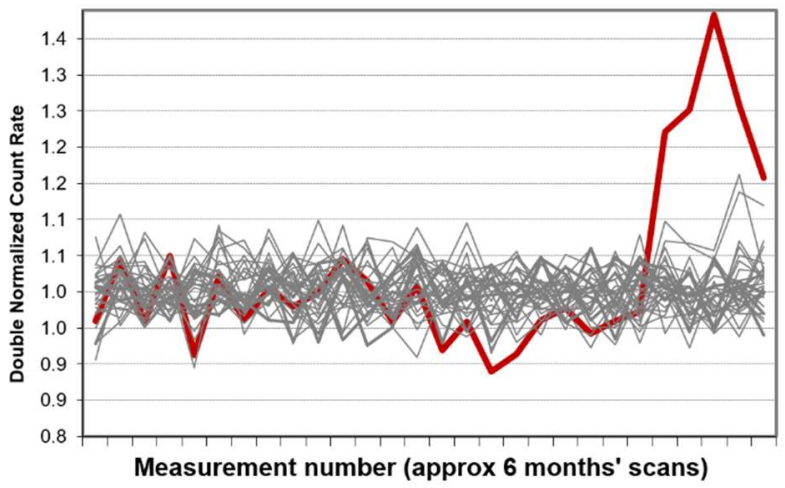

\section{Fig. 1 A representative DN plot, with defect channel highlighted}

the highlighted data series derived from a channel containing defect fuel. The channel selection process is challenging as the data has low resolution and high variance and operating experience using the existing $2 \mathrm{xN}$ system has demonstrated 
that the time taken for the channel containing the fuel defect to become distinguishable from its neighbouring channels varies considerably. Greater clarity and repeatability in this area would be beneficial.

Fuel defect detection in CANDU reactors has been the focus of some previous work. (MacDonald, Floyd, Lewis, Manzer, $\&$ Truant, 1990) summarised the current approach based on calculation and thresholding of a DN discrimination ratio, comparing count rates to background. The $2 \mathrm{xN}$ algorithm (Schmeing \& Hogg, 2005) is a direct development of this method. Other work (Livingstone, 2012) has focussed on improved online decision support for the GFP system using physical understanding of the behaviour of specific radionuclides to more quickly identify anomalous reactor parameters. To the authors' knowledge, no recent work has been undertaken to improve the DN time series analysis.

\section{GENERAL APPROACH}

The focus of this paper is on assessment of on-line DN monitoring data, with the aim of reducing the time taken between initial detection of an in-core defect to scheduling the channel for removal of the defect. The work is divided into two stages. In stage 1, an initial review of a data sample was undertaken and the potential for improvement in the existing defect localisation process was explored. In stage 2, a more extensive database was reviewed for bulk historical analysis, with the aim of visualising and identifying longer-term trends and any reactor data which may be relevant. This feature engineering process is an important step in the production of robust and explainable models. Fig. 2 outlines the development of this work.

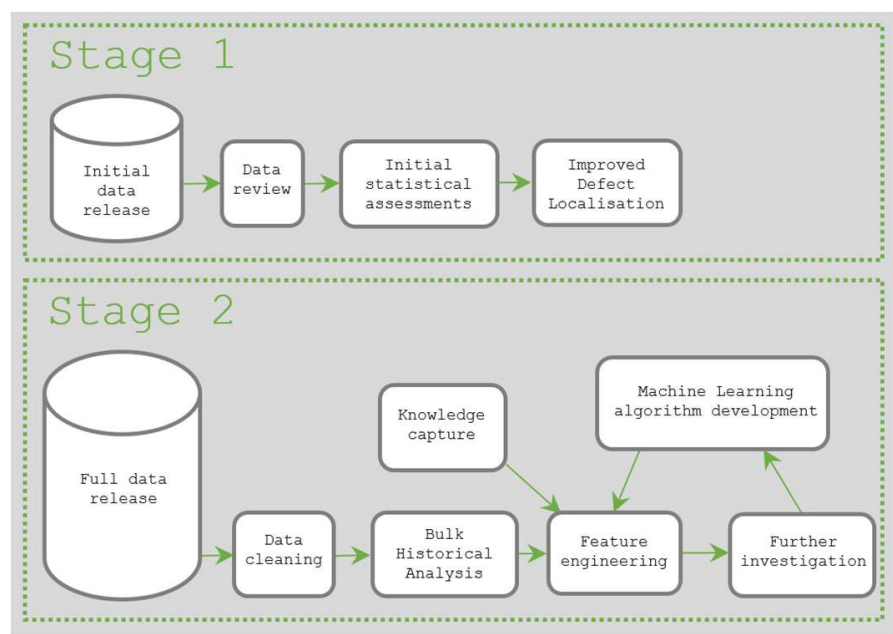

Fig. 2 General approach for the analysis of DN data

The limited initial release of DN data referred to in Fig. 2 covered several units at the Bruce Power site, for time periods around specific fuel defects of approximately one year. The full data release covered all 8 units, spanning various time periods up to 30 years. Currently, only very limited data regarding fuel defect detection and location dates is available to allow benchmarking and validation of the developed techniques.

\section{STAGE 1: IMPROVED DEFECT LOCALISATION}

Using a small representative set of data with labelled defects, preliminary assessments explored the application of alternative anomaly detection methods. For each method described in the following subsections, a series of graphs is displayed demonstrating the result of this specific analysis at various intervals approaching the time of defect localisation by the $2 \mathrm{xN}$ algorithm. We refer to this identification time point as 'D'.

\subsection{Change in Variance}

One approach was to calculate the variance of each channel from the point at which the GFP system identified an in-core fuel defect. This is shown visually in Fig. 3 for a batch of 30 channels (corresponding to one monitor), where the vertical dotted line corresponds to GFP detection date. Variance is calculated for all channels for data immediately following this point.

The results of the variance calculation are displayed graphically in Fig. 4. The defect-containing channel, $5^{\text {th }}$ from left, has been highlighted. It can clearly be seen that the variance in this channel is sufficiently distinguishable from the bulk response in the latter 2 scans. Six further instances of defect fuel data have been examined and on average it has been shown that examining the variance can distinguish the channel containing the fuel defect 2-3 scans earlier than the $2 \mathrm{xN}$ system.

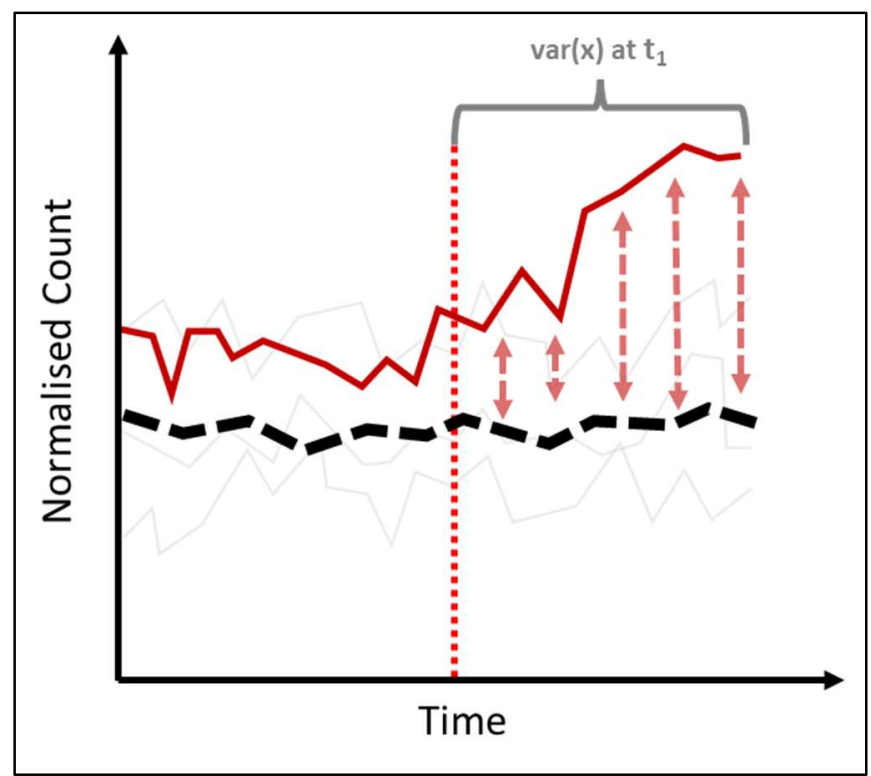

Fig. 3 Variance calculation - stylised example 


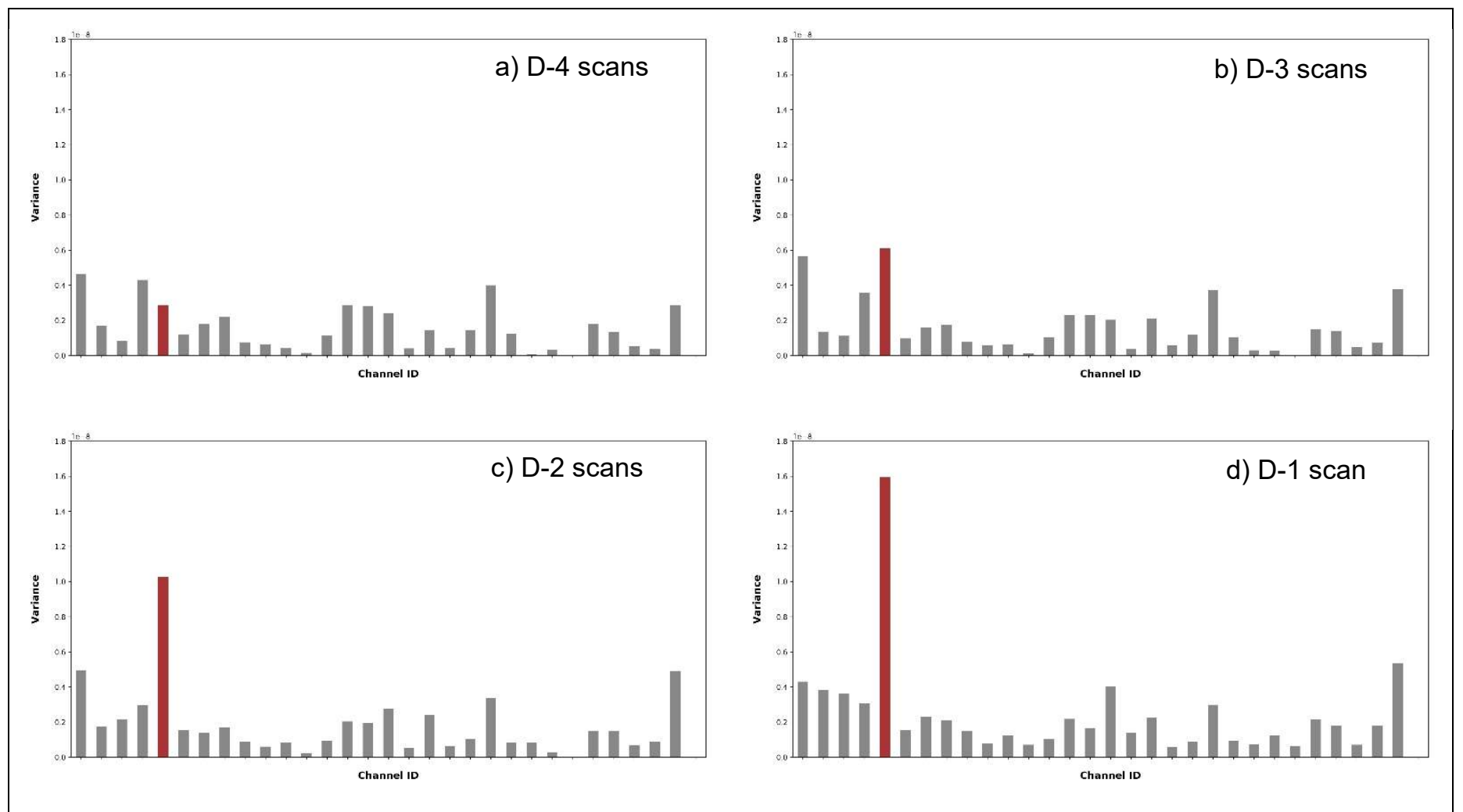

Fig. 4 Variance for all channels - results

\subsection{Change in Mean}

Another approach quantified the change in mean of the count rate for each channel, before and after detection of a defect using the GFP system. Fig. 5 shows this approach graphically.

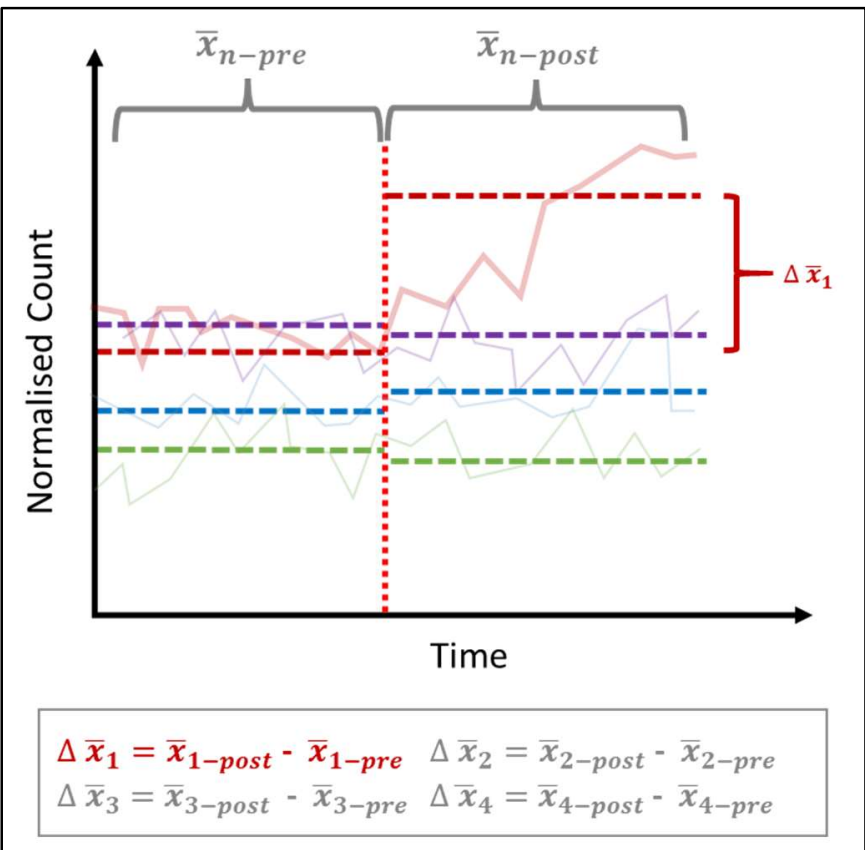

Fig. 5 Change in mean calculation - stylised example
Channels where the mean count rate decreased are dismissed on the basis that only an increased count is expected for a channel containing a defect.

This method uses the assumption that on average, the count rate should increase for a channel containing a defect and so the comparison of the mean of two similar subsequences before and after the GFP detection date should mitigate some of the noise and highlight this discrepancy. The results for this technique are shown in Fig. 6. 


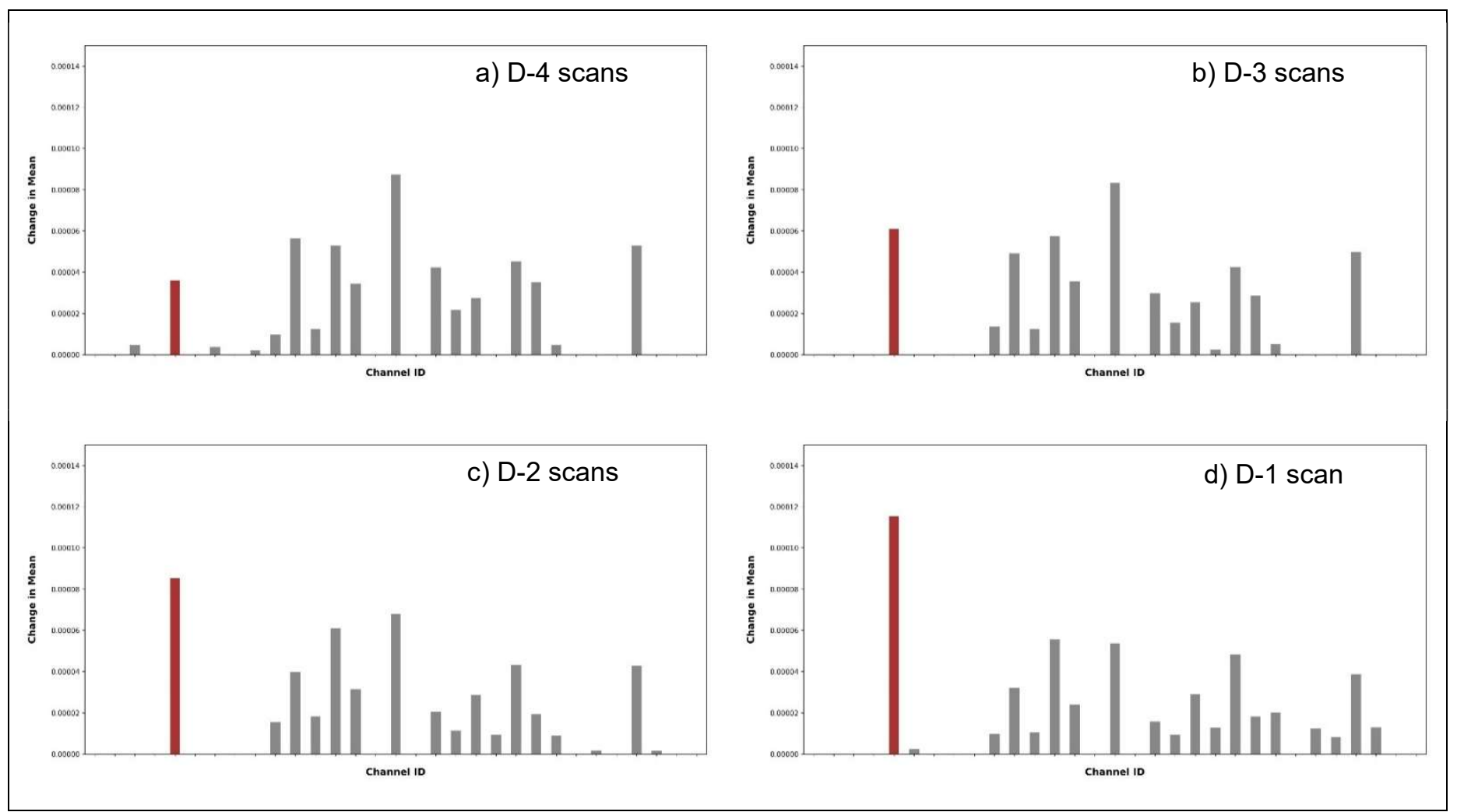

Fig. 6 Change in mean - results

As with the variance calculation method demonstrated in section 4.1, the defect channel is also visible earlier than with the $2 \mathrm{xN}$ system. Other channels do also contain relatively large positive deviations, which could be caused if the count rates in these channels prior to GFP detection were atypically low, for example. It may be possible to optimise this further by adjusting the data window size pre-GFP detection or by looking further backwards to account for any more general trends occurring in other monitors.

\subsection{Cumulative Residuals}

On the basis that small differences from the mean behaviour may not be visible due to high levels of noise, it is proposed that the cumulative effect of these differences may be more obvious. With each new scan, the final method shown calculates an array of differences between the counts and the average count in the window since the GFP detection. The cumulative sum and total sum of these differences is then calculated, with the cumulative figures finally subtracted from the total. In this way the final value is always 0 (because the final cumulative sum = total sum) and any anomalouslyhigh counts will appear above the others when plotted graphically.

A window starting at point of GFP detection could be analysed, with the exact starting point adjusted to optimise detection capability. As before, Fig. 7 displays a stylised example of this calculation process.

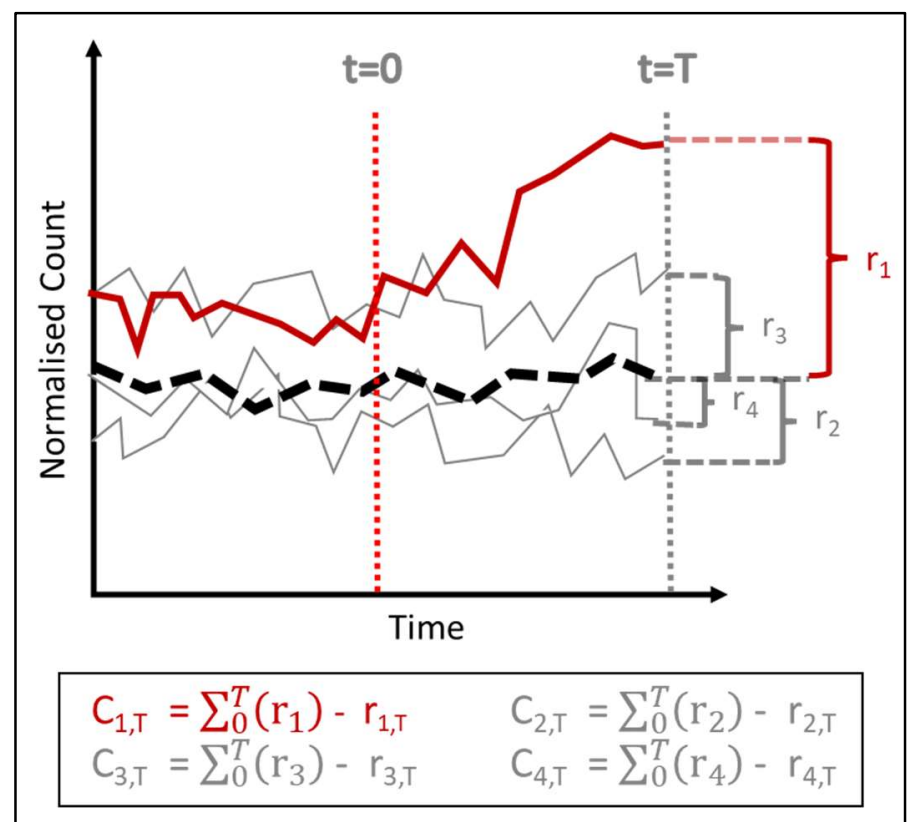

Fig. 7 Stylised example showing cumulative residual calculation

Fig. 8 displays the results of this calculation. Again, the fuel defect channel is distinguishable up to 3 scans before it is identified via the $2 \mathrm{xN}$ system. 


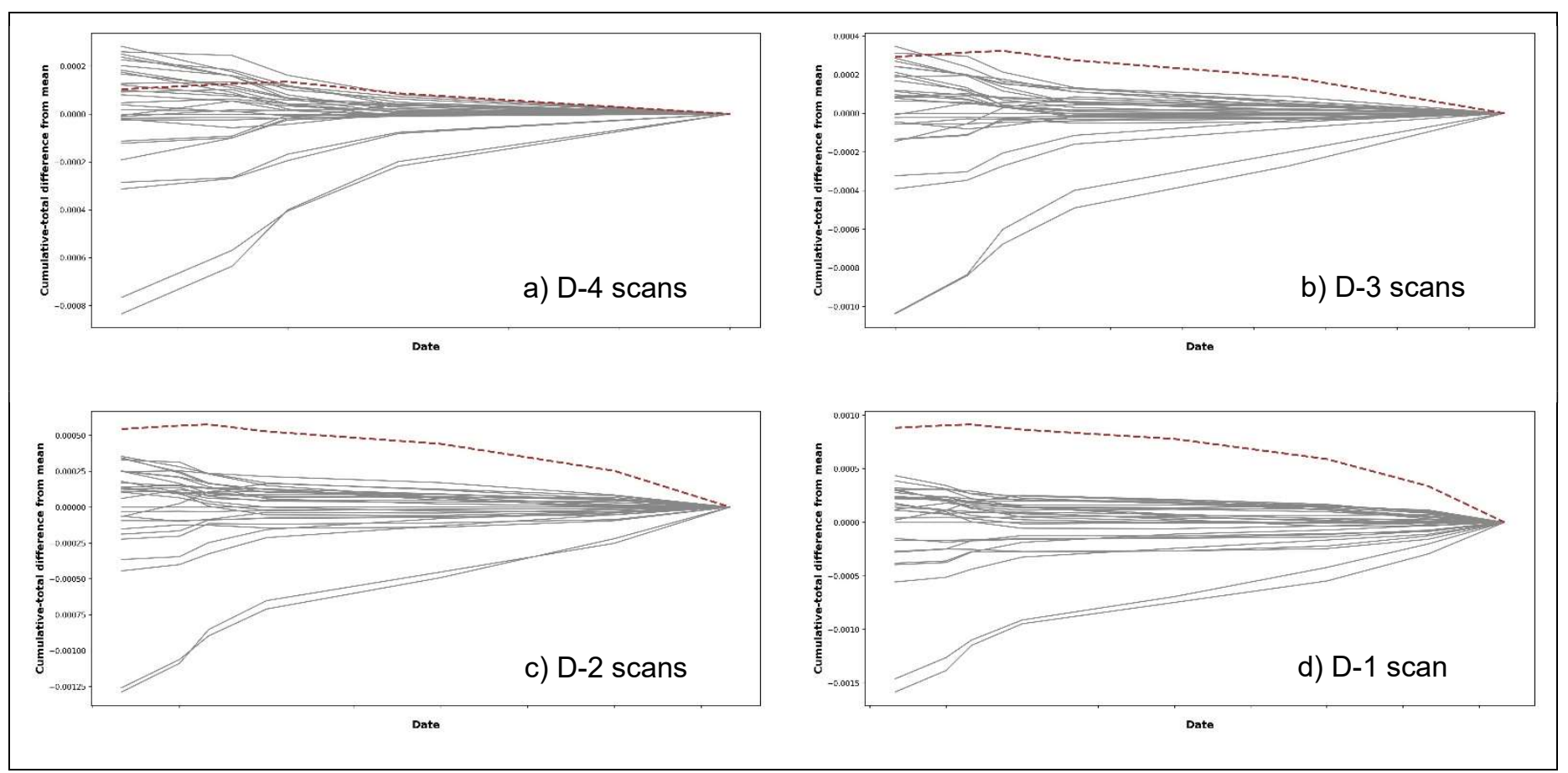

\section{Fig. 8 Cumulative Residuals}

These early visualisations provided evidence that alternative approaches to the current analysis process were possible. Three methods have been presented here which show promise for identifying anomalous channels 2-3 scans earlier than with existing methods. Further detailed analysis against other historical instances of defect fuel across different reactors is now underway in order to build a case for moving towards using one, or a combination, of these methods in conjunction with the existing double normalisation method.

\section{STAGE 2: BULK HISTORICAL ANALYSIS}

As discussed earlier, on completion of an initial review of a smaller dataset a more detailed analysis can now be made. Stage 2 of this work seeks to identify longer-term trends which can enable the incorporation of more sophisticated analytics tools. Based on the findings of the initial visualisations, a larger set of data was made available. Several years of data was parsed and extracted from the regular monitoring of 8 units. Feature information in a suitable archival form is currently limited but other trends of interest can nonetheless be identified.

As a first step, the mean, median and standard deviation of the raw data was plotted as a time series to visualize any long term trends. This is shown for one unit in Fig. 9. Each point relates to the average, median or standard deviation of a complete scan for a single monitoring hall. Overall, there appears to be a tendency for average count rates to slowly reduce over time, with step changes following extended outages. Discussion with engineers indicates that the long-term downward trend may be due to the reduction of monitor sensitivity over time when exposed to neutron flux, and the step change potentially due to recalibration and cleaning of monitors. Similar trends are visible for most other units and investigations are ongoing. 


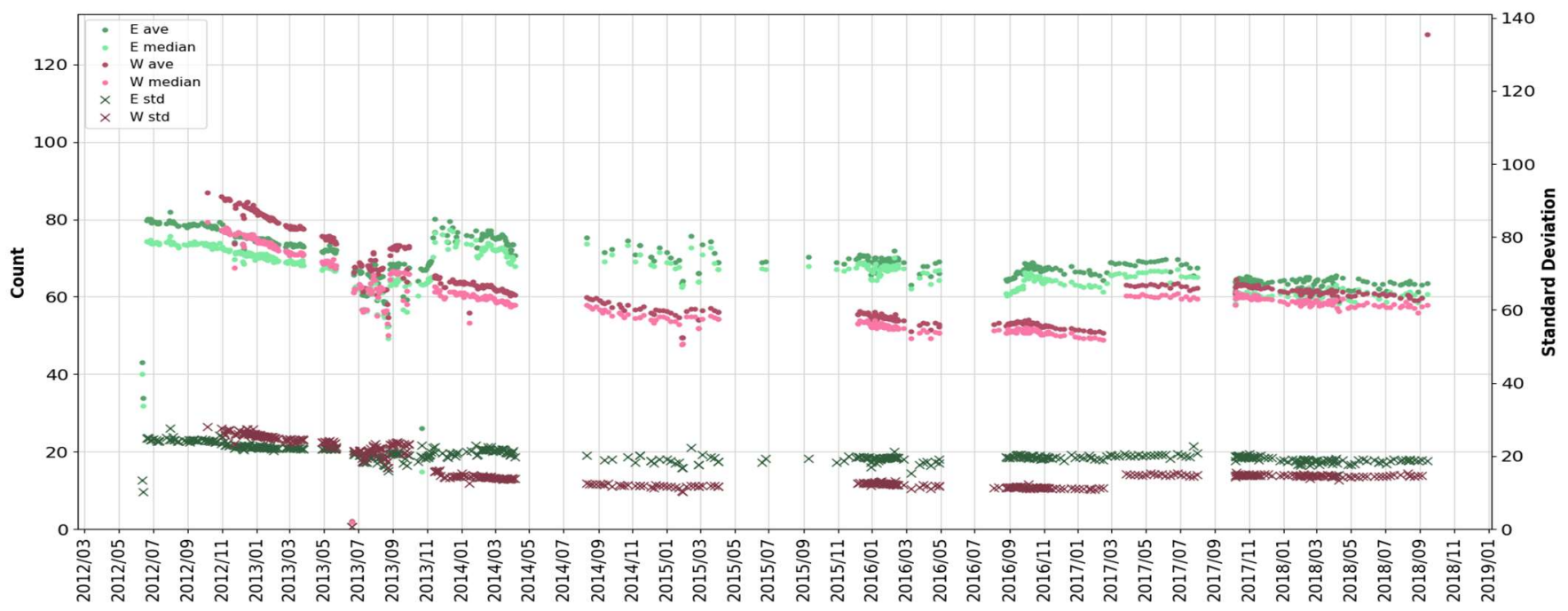

Fig. 9 Mean, Median \& Standard Deviation of raw counts

Further insight can be gained by plotting this data as a heatmap for each measurement hall. An example is shown in Fig. 10, where every vertical slice corresponds to a single scan and every horizontal slice corresponds to a single channel of the 240. In order to perform comparisons between different detectors, it is necessary to first normalise the count data. A standard scaling process was selected to produce a distribution with zero mean and unit standard deviation across the date axis for each monitor group, which allows identification of inherent activity differences between channels. The arrangement of the data is such that on the y-axis, channels are grouped by detector. Within each detector band there is graduated distribution in the normalised count from low to high, as when the system was designed there was an attempt made to ensure even distribution of low and high power channels across each detector. As with most reactor designs, central channels tend to have a higher associated power (and associated DN activity) than those on the periphery and this leads to 8 horizontally repeating patterns.

Visualising the data in this manner for this particular unit has highlighted a clear, distinctly different response in one of the detectors: a number of anomalies are visible in the $6^{\text {th }}$ horizontal band which indicates that this monitor has occasionally been causing spurious readings for several years. This is not a critical issue as errors are almost always obvious and can be quickly discarded but further investigation is ongoing with station engineers to determine the root cause of this behaviour.

It is worth noting that both Fig. 9 and Fig. 10 display approximately six years of inspection data on a single graph in a way which is not normally visualised and this has identified some previously-unseen insightful trends.

An important aspect of any analysis techniques developed is to ensure that there is an understanding of the underlying physical processes, which involved close discussion with engineers at Bruce Power. In this way, insight has been gained regarding the physical layout of the pipework connecting the reactor to measurement halls, as well as the difference in appearance of real defects compared with electronic errors. Specifically, real defects will occasionally affect adjacent coils in the measurement halls. The longer-term expected behaviour of the channel activity is also understood, where following an initial
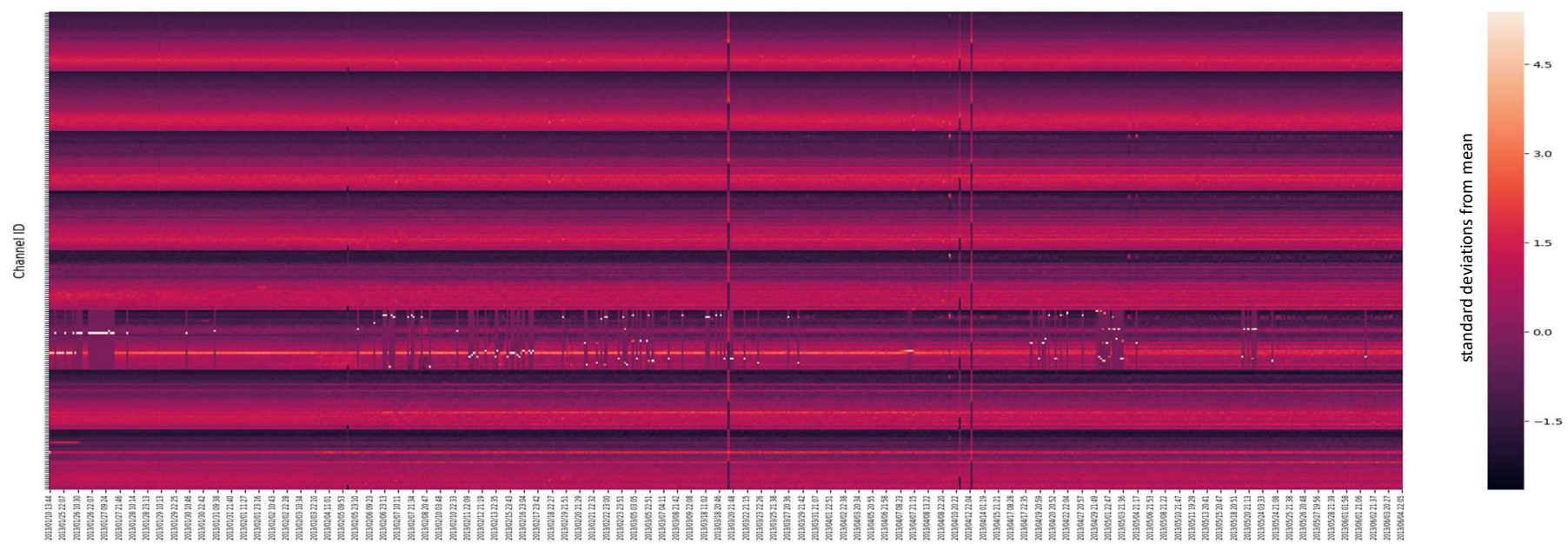

Fig. 10 Heatmap of normalised counts, scaled by monitor, coloured by standard deviations from mean 
spike in activity level in one channel, the activity level of all channels will often increase as escaped fission byproducts recirculate in the primary coolant loop. It is also suggested that any long term general behaviour of the monitors may prove to be useful in identifying anomalies in the data. The incorporation of these effects into any future model will be instrumental in the feature engineering step identified in Fig. 2.

\section{CONCLUSIONS \& FURTHER WORK}

This paper has demonstrated the additional value that can be gained from the application of modern data analytic techniques to leverage existing data sources and provide additional insights into core behaviour, demonstrated through application to delayed neutron data.

An initial review of a number of approaches toward improved DN analysis have been presented. Early work has proved promising in this regard, with some statistical analyses potentially able to identify channels containing fuel defects up to 2-3 scans earlier than the current system.

Analysis of a larger dataset has enabled longer-term trends in the data to be identified, which may identify useful inputs to more sophisticated analytics tools. Further datasets will also shortly become available and allow analysis of other factors, including but not limited to coolant temperatures, fission product levels and well-validated channel power simulation results. These inputs may allow subtler trends in the DN dataset to be isolated and allow more sensitive anomaly detection algorithms to be implemented, further reducing the time for the localisation of fuel defects.

\section{ACKNOWLEDGMENTS}

The contents, including any opinions and/or conclusions expressed, are those of the authors alone. The authors wish to thank Bruce Power for their financial and technical support of this research.

\section{REFERENCES}

AECL CANDU. (1993). Design Manual: Failed Fuel Channel Identification System. Mississauga, Ontario.

Garland, W. J., Chaplin, R., Marleau, G., Nichita, E., Rouben, B., Popov, N. K., ... Damario, D. (2014). The Essential $C A N D U$. (William J Garland, Ed.) (First). Hamilton, Ontario: University Network of Excellence in Nuclear Engineering. Retrieved from https://www.unene.ca/education/candu-textbook

IAEA. (2010). Review of Fuel Failures in Water Cooled Reactors. Retrieved from http://wwwpub.iaea.org/MTCD/Publications/PDF/Pub1445_web.p df

Lipsett, J. J., \& Tseng, C. M. (1976). Delayed-neutron systems for failed fuel location in CANDU reactors. IEEE Transactions on Nuclear Science, 23(1), 325-330. https://doi.org/10.1109/TNS.1976.4328265

Livingstone, S. J. (2012). Development of an on-line fuel failure monitoring system for CANDU reactors. Royal Military College of Canada. Retrieved from http://www.collectionscanada.gc.ca/obj/thesescanada/v ol2/002/NR83407.PDF

MacDonald, R. D., Floyd, M. R., Lewis, B. J., Manzer, A. M., \& Truant, P. T. (1990). Detecting, locating and identifying failed fuel in Canadian power reactors. Chalk River, Ontario. Retrieved from https://www.nrc.gov/docs/ML0334/ML033450220.pdf

Schmeing, H., \& Hogg, C. (2005). The Bruce B Delayed Neutron (DN) Monitoring System for Failed Fuel Detection: Twenty years of operating experience, recent improvement and planned upgrades. 